\title{
COMPUTATIONAL ANALYSIS AND PERFORMANCE EVALUATION OF REPRESENTATIVE WAFER SCALE INTEGRATION INTERCONNECTIONS
}

\author{
James C. Lyke, Jr., Captain, USAF and Edward S. Kolesar, Jr., PhD, P.E., Lt Col, USAF
}

Air Force Institute of Technology

Department of Electrical and Computer Engineering

Wright-Patterson AFB, Dayton, OH 45433-6583

\section{ABSTRACT}

A study of the electrical performance of wafer scale integration interconnections, with an emphasis on the characteristic impedance. was completed. The study was based on closedform expressions, finite difference method models, commercially available microwave circuit analysis software, and a numerical evaluation of the Telegrapher equations. Measurements were conducted on 5-inch diameter test wafers which contained numerous interconnection structures. The result revealed interesting information concerning the driving-poin impedances of the structures, the inadequacy of closed-form models for accurate estimation, and the need for more accurate modeling techniques.

\section{INTRODUCTION}

System designers continue to strive for denser and faster electronics that are beyond the capabilities of existing semiconductor fabrication processes. Just as the integrated circuit (IC) represents a technique that efficiently packages discrete electronic components, the emerging technologies that efficiently package entire groups of ICs on a common substrate are of keen interest. These technologies are commonly referred to as "wafer scale integration" (WSI), "complex hybrid microcircuits", and "multi-chip modules" (MCMs).

One challenge toward achieving a viable WSI/MCM technology involves electrical performance. A recent investigation explored the radio frequency (RF) electrical performance of WSI/MCM interconnections. ${ }^{2}$ An emphasis was placed on the characteristic impedance. Results based on closed form expressions, numerical approximations, and computer simulations were compared to the measured results.

\section{WSI/MCM DESIGN}

The WSI/MCM silicon wafer substrates (5-inch diameter) were identically patterned with 128 transmission line structures that were $10 \mathrm{~cm}$ to $120 \mathrm{~cm}$ in length. ${ }^{3}$ These substrates were provided by the Naval Ocean Systems Center (San Diego, CA), and they were fabricated by Polycon, Inc. (Phoenix, AZ). The supporting silicon wafer substrate, which served as a provisional ground plane, was coated with $\mathrm{SiO}_{2}$ $(0.5 \mu \mathrm{m}$ thick), and up to three alternating layers of aluminum conductors $(2.5 \mu \mathrm{m}$ thick) and interlevel dielectrics (8-10 $\mu \mathrm{m}$ thick). The dielectric materials were either a polyimide or benzocyclobutene (BCB).

\section{WSI/MCM INTERCONNECT TRANSMISSION LINE THEORY}

Analysis of the electrical performance of WSL/MCM interconnects requires a classification of their performance regime (lumped element, transmission line, and the full-wave regime) and application of the appropriate analytical technique. The most general and complicated analytical method involves a fullwave formulation which considers all propagation modes supported by the guided wave structures. Fortunately, for the largest class of problems of interest (sub-1 GHz digital systems with rise times greater than $200 \mathrm{psec}$ ), a full-wave analysis can usually be avoided without a significant compromise when the interconnections are shorter than $10 \mathrm{~cm}$. 'Forcing' the analysis into the transmission line regime is often referred to as the quasi-static approximation. This approximation dimensionally reduces the analysis and allows simplifying concepts, such as the characteristic impedance, to be applied.

In certain cases, further simplifications allow lumped circuit analysis techniques to be applied. One rule of thumb delineating the lumped element and transmission line regimes stipulates that a transmission line analysis should only be applied to pulser digital systems when 4

$$
t_{r}<2.5 t_{f}
$$

where $t_{\mathbf{r}}$ is the $10 \%$ to $90 \%$ rise time of a pulsed digital waveform, and $t_{f}$ is the time-of-flight delay, given as

$$
t_{f}=\ell / v_{p}
$$

where $\ell$ is the length of the interconnection, and $v_{p}$ is the propagation velocity. The propagation velocity $\left(v_{p}\right)$ is, in turn, given by 2

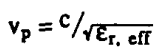

where $c$ is the velocity of light and $\varepsilon_{r, \text { eff }}$ is the effective relative dielectric constant of the medium. Using Eqs. (2) and (3), the prior 'rule of thumb' can be simplified to

$$
f>\frac{1200}{\ell \sqrt{\varepsilon_{\text {r, eff }}}}
$$

where $f$ is the clock frequency $(\mathrm{MHz})$ of a digital waveform with a $50 \%$ duty cycle and a rise time equal to one-tenth of the period, and $\ell$ is given in centimeters. This simple 'rule of thumb' illustrates the dilemma faced by designers of high-performance digital systems. As digital clock frequencies in crease, designers will seek WSI/MCM solutions to avoid transmission line effects, and thereby limit the redesign of existing systems for transmission line performance.

Transmission line theory is based on the well-known Telegrapher uyu....ns ${ }^{2}$

$$
C \frac{\partial V}{\partial \mathrm{l}}+r V+\frac{\partial I}{\partial z}=0
$$

and

$$
L \frac{\partial I}{\partial t}+R I+\frac{\partial V}{\partial z}=0
$$

255 U.S. Government work not protected by U.S. copyright. 
where $t$ is time, $z$ is the spatial variable denoting the direction of propagation, and $\mathrm{R}, \mathrm{L}, \mathrm{G}$, and $\mathrm{C}$ are the distributed series resistance, inductance, shunt conductance, and capacitance, respectively, expressed on a per unit length basis.

The phasor solution of Eqs. (5) and (6) can be expressed as

$V(z)=V_{+} \exp (-\xi z)+V \cdot \exp (\xi z)$

and

$$
I(z)=\frac{V_{+}}{Z_{o}} \exp (-\xi z)-\frac{V_{-}}{Z_{o}} \exp (\xi z)
$$

where $\xi$ is the complex propagation constant, $\mathrm{V}_{+}$and $\mathrm{V}_{\text {. are }}$ the amplitudes of the forward (incident) and backward (reflected) voltage wave components, and $\mathrm{Z}_{\mathrm{o}}$ is the characteristic impedance, sometimes defined as 2

$$
Z_{0}=\sqrt{\frac{(R+j \omega L)}{(G+j \omega C)}}
$$

where $\omega$ is the angular frequency $(\mathrm{rad} / \mathrm{sec})$. An altemate definition of the characteristic impedance for a lossless line is

$$
z_{o}=1 / c \sqrt{C_{o} C}
$$

where $C$ is the capacitance per unit length $0_{i}^{-} \dot{\text { is }}$ structure, and $C_{0}$ is the capacitance per unit length of the same structure when the dielectric material is replaced with the free space condition.

\section{CHARACTERISTIC IMPEDANCE DETERMINATION}

For rectangular transmission line structures, many closed form expressions for $Z_{0}$ have been developed. For more geometrically complicated structures, numerically based analytic methods involving Eq. (10) are necessary. As a part of the investigation, the accuracy of various closed form expressions for $Z_{0}$ reported in the literature were compared.

\section{EMBEDDED MICROSTRIP}

One expression was found for the per unit length capacitance of an embedded microstrip that is based upon the direct conformal transformation of a structure with non-zero thickness. For the case of a single conductor with non-zero width (w), thickness (t), positioned at height (h) above a single ground plane, and embedded in a homogeneous dielectric of thickness (b), the per unit length capacitance is estimated to be 5

$$
C=\frac{2 \varepsilon_{0} \varepsilon_{\mathrm{r}, \text { eff }}}{\pi} \ln \left(\frac{2 R_{\mathrm{b}}}{\mathrm{R}_{\mathrm{a}}}\right)
$$

where

$$
\begin{aligned}
& \ln R_{a}=-1-\frac{\pi w}{2 h}-A_{1}-\ln [(p-1) / 4 p] \\
& A_{1}=[(p+1) / \sqrt{p}] \cdot \tanh ^{-1}(1 / \sqrt{p}) \\
& R_{b}=\eta+[(p+1) / 2 \sqrt{p}] \ln \Delta \\
& \eta=\sqrt{p}\left[\frac{\pi w}{2 h}+A_{2}\right]-2 \tanh ^{-1}(1 / \sqrt{p}) \\
& A_{2}=[(p+1) / 2 \sqrt{p}] \cdot A_{3}
\end{aligned}
$$

$$
\begin{aligned}
& A_{3}=[1+\ln (4 /(p-1))] \\
& \Delta=\max (\eta, p) \\
& p=2 b^{2}-1+\sqrt{\left(2 b^{2}-1\right)^{2}-1} \\
& b=1+(t / h) .
\end{aligned}
$$

Eq. (11) is reported to be accurate to within one percent when $(w / h) \geq 1$. With the capacitance known, the characteristic impedance can be found using Eq. (10).

\section{STRIPLINES}

Many expressions were found for stripline configurations. The simplest closed form expression for the $Z_{0}$ of striplines with zero thickness is given by ${ }^{2}$

$$
Z_{0}=\frac{30 \pi K(k)}{\sqrt{\varepsilon_{\mathrm{r}}} K\left(\mathbf{k}^{\prime}\right)}
$$

where $K(k)$ is the complete elliptic integral of the first kind, with $\mathbf{k}$ and $\mathbf{k}$ ' defined as

$$
k=\operatorname{sech}[\pi w /(2 b)] \quad k^{\prime}=\operatorname{tanh!} \pi /(2 b)
$$

where (w) is the conductor width, and (b) is the ground plane separation distance.

Wheeler's approximation for single striplines of non-zero thickness is slightly more involved and is given by 2

$$
Z_{0}=\frac{30}{\sqrt{\varepsilon_{r}}} \ln \left\{1+\frac{4}{\pi} \frac{b t}{w^{\prime}} \eta\right\}
$$

where

$$
\begin{aligned}
& \eta=\frac{8}{\pi} \frac{(b-t)}{w^{\prime}}+\sqrt{\left(\frac{8}{\pi} \frac{(b-t)}{w^{\prime}}\right)^{2}+6.27} \\
& \frac{w^{\prime}}{(b-t)}=\frac{w}{(b-t)}+\frac{\Delta w}{(b-t)} \\
& \frac{\Delta w}{(b-t)}=\frac{x}{\pi(1-x)}\left[1-\frac{1}{2} \ln A_{1}\right] \\
& A_{1}=\left[\frac{x}{(2-x)}\right]^{2}+\left[\frac{0.0796 x}{(w / b)+1.1 x}\right] \\
& m=2\left[1+\frac{2}{3(1-x)}\right] \\
& x=\left(\frac{x}{b}\right) .
\end{aligned}
$$

This expression is accurate to within 0.5 percent when $w^{\prime} /(b-t)$ is less than 10

The finite difference method (FDM) was selected as an alternate method for the analysis of WSI/MCM interconnections. To implement this approach, two-dimensional transverse cross-sections of the candidate WSIMCM structures were modeled as shown in Fig. 1 using a discretized grid in the $x$ and $y$ dimensions with truncated sidewalls. The FDM solution for the electrostatic potential (V) is given by

$$
\Delta \mathrm{V}=0
$$

The nodes (grid points in Fig. 1) are mathematically formulated with simple iterative expressions. ${ }^{6}$ Given the potential distribution, the characteristic impedance is found from ${ }^{2}$ 


$$
Z_{0}=\sqrt{\frac{\mu}{\bar{\varepsilon}_{0} \varepsilon_{\mathrm{r}}}} \frac{\int_{\Gamma_{\mathrm{i}}} \overline{\mathrm{E}} \cdot \overline{\mathrm{d} \ell}}{\int_{\gamma_{\mathrm{i}}}(\overline{\mathrm{E}} \cdot \hat{\mathrm{n}}) \overline{\mathrm{d} \ell}}
$$

where the contour integrals $\Gamma_{\mathrm{i}}$ and $\gamma_{\mathrm{i}}$ are shown in Fig. 2. The electric field $(E)$ can be readily computed via a simple firstorder finite difference calculation involving the potential values.

The characteristic impedance was also calculated with the EESoft, Inc. LINECALC ${ }^{\mathrm{TM}}$ software. Interconnection structures were also modeled using the EESoft LIBRA ${ }^{\mathrm{TM}}$ program.

\section{MEASUREMENT TECHNIOUF}

This research effort also focused on measuring the characteristic impedance of selected transmission line structures on the Polycon fabricated wafers. In addition, coupling and interconnection series resistance measurements were accomplished.

The characteristic impedance measurements were performed on the test structures with an RF impedance analyzer at frequencies spanning 1-700 MHz. Microprobing was performed with coariui needle probes and calibrated cabling. Short-circuit $\left(Z_{s c}\right)$ and open-circuit $\left(Z_{o c}\right)$ driving point impedances were used in a common relationship to obtain the characteristic impedance $\left(Z_{0}\right)$ :

$$
\left|Z_{o}\right|=\sqrt{\left|Z_{o c}\right| \cdot\left|Z_{s c}\right|}
$$

and

$$
\operatorname{Phase}\left(Z_{o}\right)=\frac{1}{2}\left[\operatorname{Phase}\left(Z_{o c}\right)+\operatorname{Phase}\left(Z_{s c}\right)\right] \text {. }
$$

\section{DISCUSSION}

Two interccinection structures were modeled analytically using the parameters in Table I with the same closed form expressions. FDM simulations were also implemented. As predicted by Eq. (16), the product of the open- and short-circuit driving point impedances is a constant, but the actual measurements revealed a frequency-dependent behavior (Fig. 3). This behavior was simulated analytically by extracting the $R$, $L, G$, and $C$ parameters of the associated transmission line structure and applying Eqs. (5) and (6) at a set of discrete frequencies. In this simulation, the leakage conductance $(G)$ was assumed to be zero, and the series loss resistance (R) was estimated from

$$
R=\rho \ell / A
$$

where $\rho$ is the resistivity of the aluminum conductor $\left(2.8 \times 10^{-6}\right.$ ohm-cm), and $\ell$ and $A$ are its length and transverse cross-sectional area, respectively. The capacitance (C) was extracted from the rLivi simulation, and the inductance (L) was obtained from ${ }^{2}$

$$
\mathrm{L}=\mu \mathrm{F} \text {. }
$$

Small amounts of random noise and the series loss of the interconnections were introduced into the simulation to more ac- curately model the behavior experienced during the measurements (Fig. 3).

A simple technique was developed to estimate a single-valued $\mathrm{Z}_{0}$ from the driving point measurements by applying the same FDM simulation used to achieve the electrostatic and frequency-dependent values. This technique determined a crossing frequency ( $f_{\text {cross }}$ ) where the $Z_{0}\left(f_{\text {cross }}\right)$ of the frequencydependent simulation crossed or closely approached the value (to within $10^{-3}$ ) of $Z_{0}$ obtained from the electrostatic FDM simulation. The value of the driving point measurement of $Z_{0}=Z_{0}\left(f_{c r o s s}\right)$ was then obtained using linear extrapolation.

The set of analytic and measured data for the characteristic impedance of the embedded microstrip and stripline structures is presented in Table II. For the stripline structure, the measured value of $\mathrm{Z}_{0}$ was slightly larger than most of the analytically calculated values. For the embedded microstrip, the measured value of $Z_{0}$ was significantly larger than all of the calculated values. Three limitations affected the accuracies of the models evaluated: (1) the assumption of infinite conductivity of the silicon substrate so that it could be modeled as a ground plane, (2) a $20 \%$ uncertainty in the physical dimensions of the structures, and (3) the assumption of solid ground planes (meshed ground planes were common).

\section{CONCLUSIONS}

This research effort explored the characteristic impedance parameter and its relevance to WSI/MCM interconnections. Understanding their transmission line performance is important, since operation in this regime can have a profound effect upon system performance.

The utility of closed form expressions for this class of interconnections is limited. Practical systems include multiple layers of interconnects that are not electrically isolated, and conse quently, numerical analysis techniques are required for accurate assessments. It is important to recognize that, in attempting to avoid the transmission line regime, the concept of $Z_{0}$ has little, if any, utility. When $Z_{0}$ is important, the CAD systems, which use oversimplified estimates of $Z_{0}$ as a basis for design, are necessarily conservative. Thus, the ability to perform accurate and efficient aralyses for 'real' systems is needed, especially as more aggressive and complicated WSI/MCM system designs are pursued.

\section{REFERENCES}

1. MIL-H-38534, General Specification for Complex Hybrid Microcircuits. Military Specificarion, August 1989.

2. J.C. Lyke, "Silicon Hybrid Wafer Scaie Integration Interconnect Evaluation," MS Thesis, Air Force Institute of Technology (AFIT/GE/ENG/89D-27), WPAFB, OH, 1989.

3. J.C. Lyke and E.S. Kolesar, "An Analysis of the Electrical Performance of Wafer Scale Integration Interconnections," Connection Technology, vol. 6, pp. 41-45, 1991.

4. H.B. Bakoglu, Circuits, Interconnections, and Packaging for VLSI. Reading, MA: Addison-Wesley Publishing Company, 1990.

5. W.H. Chang, "Analytical IC Metal-Line Capacitance For mulas," IEEE Transactions on Microwave Theory and Techniques, vol. MTT-24, pp. 608-611, 1976.

6. H.E. Green, "The Numerical Solution of Some Important Transmission-Line Problems," IEEE Transactions on Microwave Theory and Techniques, vol. MTT-13, pp. 676-692 1965. 
Table I. Parameters USEd to Calculate the CHARACTERISTIC IMPEDANCE $\left(\mathrm{Z}_{\mathrm{O}}\right)$.

\begin{tabular}{|c|c|c|}
\hline \multirow[b]{2}{*}{ Structure Eeature } & \multicolumn{2}{|c|}{ Structure Type } \\
\hline & Srripline & $\frac{\text { Embedded }}{\text { Microstrip }}$ \\
\hline Conductor Material & Aluminum & Aluminum \\
\hline Intermetal Dielectric & BCB & Polyimide \\
\hline Dielectric Constant & 2.75 & 3.4 \\
\hline Conductor Width $(\mu \mathrm{m})$ & 10 & 25 \\
\hline $\begin{array}{l}\text { Ground Plane Type } \\
\text { Height Above Ground }\end{array}$ & Solid & Mesh \\
\hline $\begin{array}{l}\text { Plane }(\mu \mathrm{m}) \\
\text { Distance Between }\end{array}$ & 8 & 6 \\
\hline $\begin{array}{l}\text { Conductors }(\mu \mathrm{m}) \\
\text { Distance Between }\end{array}$ & 38 & 22 \\
\hline Ground Planes ( $\mu \mathrm{m})$ & 26.5 & N/A \\
\hline
\end{tabular}

Table II. ANALYTIC AND MEASURED VALUES OF THE CHARACTERISTIC IMPEDANCE $\left(Z_{O}\right)$ FOR TWO REPRESENTATIVE W AFER SCALE INTEgRaTION MULTI-ChIP MODULE INTERCONNECTIONS.

\begin{tabular}{lll}
\hline $\begin{array}{l}\text { Analytic and Measured } \\
\text { Categories }\end{array}$ & $\begin{array}{l}\text { Stripline } \\
\mathrm{Z}_{\mathrm{O}} \text { (ohms) }\end{array}$ & $\begin{array}{l}\text { Embedded } \\
\text { Microstrip } \\
\mathrm{Z}_{\mathrm{O}} \text { (ohms) }\end{array}$ \\
\hline Microstrip Formula & 77.82 & 33.83 \\
Equation (13) & 66.90 & 29.36 \\
Equation (15) & 71.85 & 29.21 \\
Equation (16) & 61.65 & 26.02 \\
LINECALC & 57.41 & 25.63 \\
FDM & 68.21 & 27.96 \\
Measured & 73.58 & 45.85 \\
\hline
\end{tabular}

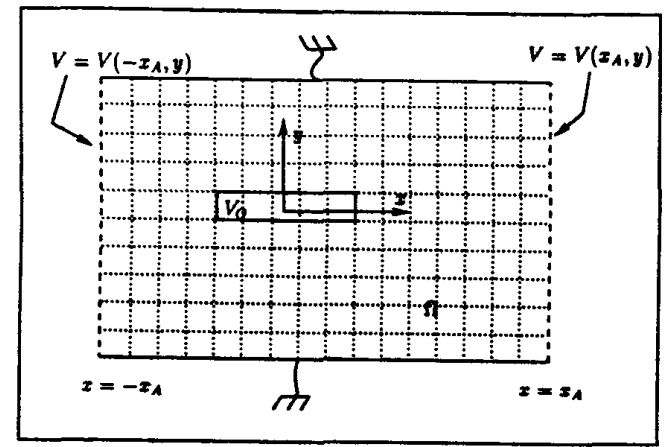

Figure 1. Finite Difference Method (FDM) Formulation -. Schematic Representation of a Stripline Transverse CrossSection.

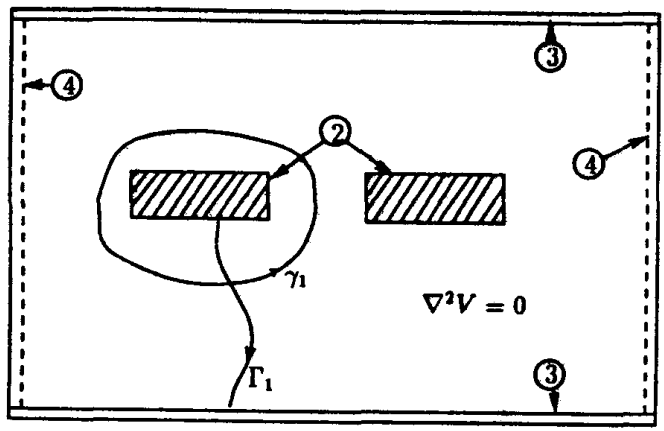

Figure 2. Schematic Representation of the Characteristic Impedance Problem Formulation Illustrating the: (1) Interior, (2) Transmission Line Conductors, (3) Ground Planes, and (4) Sidewalls.

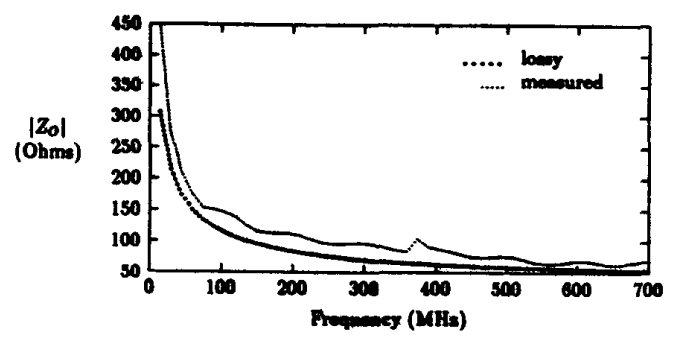

Figure 3. Magnitude of the Characteristic Impedance of an Edge-Coupled WSLMCM Stripline Interconnect as a Function of Frequency. 